\title{
Editorial
}

\author{
Dirk Slock \\ Mobile Communications Department, EURECOM Institute, 2229 route des Cretes, BP 193, 06904 Sophia Antipolis Cedex, France \\ Email: dirk.slock@eurecom.fr
}

\section{Vahid Tarokh}

Department of EECS MIT, Cambridge, MA 02139, USA

Email:vahid@mit.edu

\author{
Xiang-Gen Xia \\ Department of ECE, University of Delaware, Newark, DE 19716, USA \\ Email:xxia@ee.udel.edu
}

Space-time coding for multiple transmit and multiple receive antenna systems has recently attracted considerable attention in the wireless communications area. It has been shown theoretically that for certain channels the capacity of a multiple antenna system increases linearly with the number of antennas, which implies that multiple antenna systems have huge potential applications in broadband wireless communications. In a multiple antenna system, a key building block is multiple antenna coding and modulation, called space-time coding. In single antenna systems, the coding and modulation only deals with one-by-one complex symbols or symbol sequences. However, in multiple antenna systems, the spacetime coding deals with complex symbol matrices or matrix sequences.

In space-time coding, space-time code design is one of the most important topics. Unlike the conventional single antenna AWGN channel, the multiple antenna channel transfer function is a matrix instead of a scalar, even in the simplest propagation scenario. So, the space-time code design is more challenging than in single antenna systems. Concerning a multiple antenna channel, that is, a multiinput multi-output (MIMO) system, two cases can arise: the MIMO channel can be known or unknown at the receiver. Since the channel is a complex matrix, signal processing may play an even more important role than it does in a single antenna system. When we deal with space-time matrices, the code size may be huge and therefore fast decoding algorithms are critically important. As a result, space-time coding has become one of the most active research areas in wireless communications. The EURASIP JASP has dedicated two special issues to this subject. The first issue has 11 papers that spread over space-time code designs, decoding methods for spacetime coded transmissions, and MIMO systems.
The first three papers are on space-time code designs. The paper by S. M. Haas, J. H. Shapiro, and V. Tarokh considers space-time coding issues for wireless optical channels. The space-time codes are used to overcome turbulence-induced fading in an atmospheric optical heterodyne communication system. They propose a new criterion for the spacetime code design for such channels. The paper by Z. Safar and K. J. R. Liu considers a systematic space-time trellis code design method by jointly considering diversity and coding advantages. They propose to directly assign channel symbols to transmit antennas at different states by exploiting the properties of the state transitions in the trellis. The paper by D. Tujkovic, M. Juntti, and M. Latva-aho considers spacetime turbo code designs. They propose a design method for recursive space-time trellis codes and parallel-concatenated space-time turbo coded modulation that can be applied to an arbitrary existing space-time trellis code. This method enables a large, systematic increase in coding gain while preserving the maximum transmit diversity gain and bandwidth efficiency property of the space-time trellis code considered.

The next six papers are on decoding methods of spacetime coded transmissions. The paper by B. A. Bjerke and J. G. Proakis considers the equalization of a multiple transmit and receive antenna wireless system. They consider uncoded, convolutionally coded, turbo coded MIMO channels, and also precoded MIMO channels. The paper by E. Erez and M. Feder proposes new decoding techniques for diversity channels employing space-time codes when the channel coefficients are unknown to both transmitter and receiver. They propose an efficient implementation of the generalized maximum-likelihood ratio test algorithm and an energy weighted decoder. The paper by S. K. Jayaweera and H. V. Poor considers low complexity multiuser detection 
methods for space-time coded synchronous multiple-access systems in the presence of independent Rayleigh fading. They show that optimal space-time codes designed for single user channels can still provide full diversity in the multiuser channel. The paper by J. Liu, J. Li, and E. G. Larsson considers a differential space-time block code modulation for DS-CDMA systems. They propose three demodulation schemes, namely a differential space-time block code Rake receiver, a differential space-time block code deterministic receiver, and a differential space-time block code deterministic de-prefix receiver. The paper by Y. Liu and X. Wang proposes a space-time differential decoding technique based on multiple-symbol detection and decision-feedback by making use of the second-order statistics of the fading. The paper by J. Zhang and P. M. Djurić considers joint estimation and decoding of space-time trellis codes by employing a new emerging tool in statistical signal processing, sequential importance sampling.

The final two papers are on MIMO systems. The paper by A. Grant considers information theoretic properties of flat fading channels with multiple antennas when perfect channel knowledge at the receiver is assumed. Expressions for maximum information rates and outage probabilities are derived. It is shown that the use of orthogonal modulating signals is asymptotically optimal in terms of information rate. The paper by T.-L. Tung and K. Yao considers the problem of channel estimation and optimal power allocation for a multiple-antenna OFDM system. They develop a least-squares channel estimation approach, derive a performance bound, investigate the optimal training sequence for initial channel acquisition, and derive the optimal power allocation solution that maximizes the bandwidth efficiency under power and QoS constraints.

\section{Dirk Slock Vahid Tarokh Xiang-Gen Xia}

Dirk Slock received the engineer's degree from the University of Gent, Belgium in 1982. In 1984 he was awarded a Fulbright scholarship for Stanford University, USA, where he received his M.S. in Electrical Engineering, M.S. in Statistics, and Ph.D. in Electrical Engineering in 1986, 1989, and 1989, respectively. While at Stanford, he developed new fast recursive least-squares algorithms for adaptive filtering. In 1989-91,

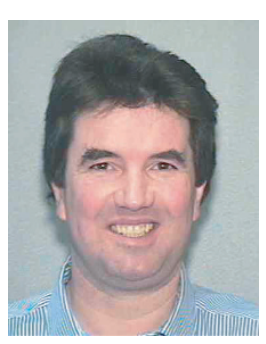
he was a member of the research staff at the Philips Research Laboratory, Belgium. In 1991, he joined the Eurecom Institute where he is now Associate Professor. At Eurecom, he teaches statistical signal processing and speech coding for mobile communications. His research interests include DSP for mobile communications: antenna arrays for (semi-blind) equalization/interference cancellation and spatial division multiple access, space-time processing and audio coding. More recently, he has been focusing on receiver design, downlink antenna array processing, and speech coding for third generation systems, and introducing spatial multiplexing in existing wireless systems. He received one best journal paper award from the IEEE-SP and one from EURASIP in 1992. He is the coauthor of two IEEE-Globecom 98 best student paper awards. He has been an Associate Editor for the IEEE-SP Transactions.

Vahid Tarokh received his Ph.D. degree in Electrical Engineering from the University of Waterloo, Ontario, Canada in 1995. From August 1995 to May 1996, he was employed by the Coordinated Science Laboratory, of the University of Illinois UrbanaChampaign, as a visiting Professor. He then joined the AT\&T Labs-Research, where he was employed as a Senior Member of Technical Staff, Principal Member of Technical

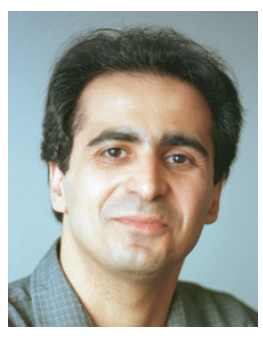

Staff, and the Head of the Department of Wireless Communications and Signal Processing until August 2000. In the fall of 2000, Dr. Tarokh joined the Department of Electrical Engineering and Computer Sciences of MIT as an Associate Professor, where he is currently employed. Dr. Tarokh received numerous awards including the 1987 Gold Tablet of The Iranian Math Society, the 1995 Governor General of Canada's Academic Gold Medal, the 1999 IEEE Information Theory Society Prize Paper Award, (jointly with A. R. Calderbank and N. Seshadri), and more recently the 2001 Alan T. Waterman Award.

Xiang-Gen Xia received his B.S. degree in mathematics from Nanjing Normal University, Nanjing, China, and his M.S. degree in mathematics from Nankai University, Tianjin, China, and his Ph.D. degree in Electrical Engineering from the University of Southern California, Los Angeles, USA in 1983, 1986, and 1992, respectively. He was a Lecturer at Nankai University, China during 1986-1988, a Teaching Assistant at University of Cincin-

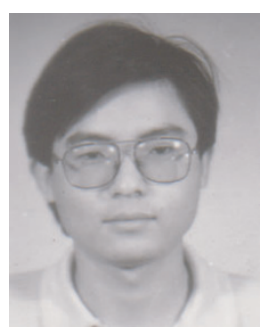
nati, USA during 1988-1990, a Research Assistant at the University of Southern California, USA during 1990-1992, and a Research Scientist at the Air Force Institute of Technology during 1993-1994. He was a Senior/Research Staff Member at Hughes Research Laboratories, Malibu, California, during 1995-1996. In September 1996, he joined the Department of Electrical and Computer Engineering, University of Delaware, Newark, Delaware, USA, where he is currently an Associate Professor. His current research interests include communication systems including equalization and coding; SAR and ISAR imaging of moving targets, wavelet transform and multirate filterbank theory and applications; time-frequency analysis and synthesis; and numerical analysis and inverse problems in signal/image processing. Dr. Xia has over 80 refereed journal articles published, and 4 U.S. patents awarded. He is the author of the book "Modulated Coding for Intersymbol Interference Channels" (New York, Marcel Dekker, 2000). Dr. Xia received the National Science Foundation (NSF) Faculty Early Career Development (CAREER) Program Award in 1997, the Office of Naval Research (ONR) Young Investigator Award in 1998, and the Outstanding Overseas Young Investigator Award from the National Nature Science Foundation of China in 2001. He also received the Outstanding Junior Faculty Award of the Engineering School of the University of Delaware in 2001. He is currently an Associate Editor of the IEEE Transactions on Mobile Computing, the IEEE Transactions on Signal Processing and the EURASIP Journal of Applied Signal Processing. He is also a Member of the Signal Processing for Communications Technical Committee in the IEEE Signal Processing Society. 